\title{
Central insulin regulates eating behaviour
}

\section{C4}

...food value

signals were

decreased in

participants

with normal

insulin

sensitivity...
Previous work in animal models has suggested that insulin could be involved in hedonic eating via actions on the dopaminergic reward system. A new study, published in Nature Communications, demonstrates that central insulin has similar effects in humans.

"These recent animal data are fascinating, but it was important to see whether these findings could be transferred to the human brain, and how this effect depends on individuals' insulin sensitivity," explains corresponding author Stefanie Brassen. To answer these questions, the

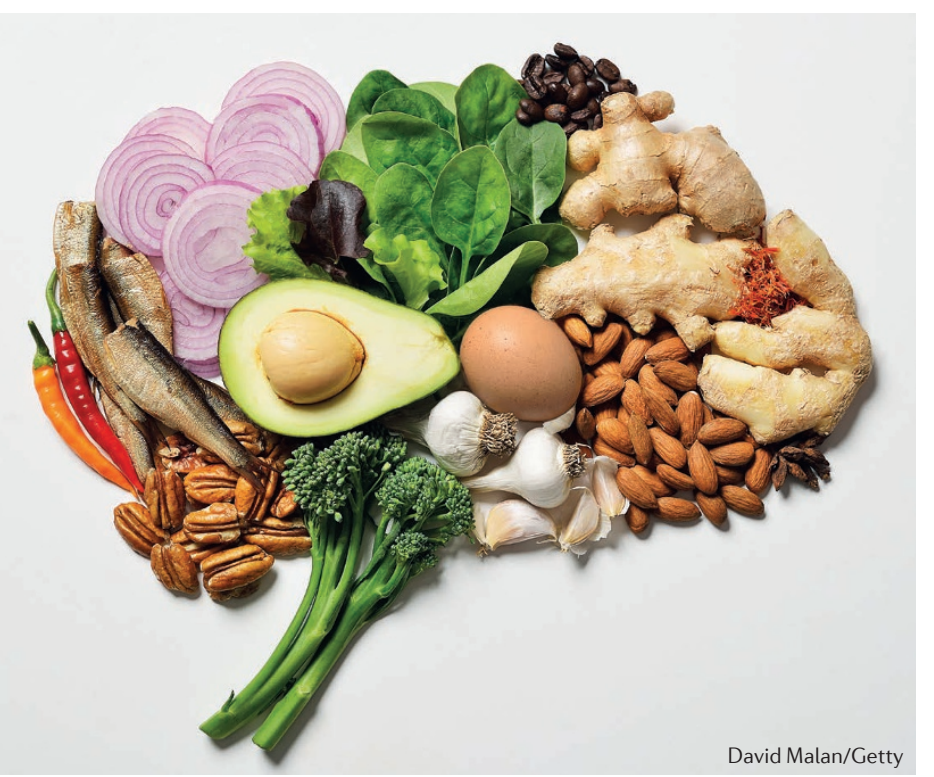

researchers enrolled 48 nondiabetic individuals who were overweight or normal weight. The participants were split into two groups based on their insulin sensitivity: normal insulin sensitivity or insulin resistance.

Following an overnight fast, the participants underwent two functional MRI procedures, separated by at least a week. Before each procedure participants were given either a placebo or intranasal insulin. Once in the scanner, participants were shown a series of images of food and non-food items and were asked to press a button to indicate either 'I like this' or 'I do not like this', which was followed by a four-point rating system to show how much they liked or disliked the item.

The researchers found that after administration of intranasal insulin, participants with normal insulin sensitivity reported reduced ratings of food liking, compared with when these participants received the placebo. By contrast, intranasal insulin administration was associated with a nonsignificant trend for increased food preference scores in the participants with insulin resistance.

Next, the researchers assessed the effects of intranasal insulin administration in areas of the brain known to be involved in reward signalling. In the bilateral nucleus accumbens and the left ventral tegmental area, food value signals were decreased in participants with normal insulin sensitivity following intranasal administration of insulin. Interestingly, the signals were increased in participants with insulin resistance under the same conditions. Thus, the authors suggest that administration of insulin decreases the reward value of foods via modulation of the dopaminergic reward system. "Our findings highlight an important mechanism by which the satiety hormone insulin is involved in normal and pathological eating behaviour," says Brassen. "The fact that we already see dysfunctions in insulinergic action in prediabetic, insulin-resistant individuals highlights the clinical relevance of this finding."

Brassen and her colleagues are now trying to determine whether the amount of weight lost after a 3-month caloric restriction diet can be used to predict normalization of neural insulin function. "It is also important to note that the effects of insulin on the reward system are probably more complex than we have shown in this first study, for example, there are probably complex interactions with other peptides, including leptin and ghrelin," concludes Brassen.

Claire Greenhill

ORIGINAL ARTICLE Tiedemann, L. J. et al. Central insulin modulates food valuation via mesolimbic pathways. Nat. Commun. 8, 16052 (2017) 